\title{
Multilingual Speech Translation with Unified Transformer: Huawei Noah's Ark Lab at IWSLT 2021
}

\author{
Xingshan Zeng, Liangyou Li, Qun Liu \\ Huawei Noah's Ark Lab \\ \{zeng.xingshan, liliangyou, qun.liu\}@huawei.com
}

\begin{abstract}
This paper describes the system submitted to the IWSLT 2021 Multilingual Speech Translation (MultiST) task from Huawei Noah's Ark Lab. We use a unified transformer architecture for our MultiST model, so that the data from different modalities (i.e., speech and text) and different tasks (i.e., Speech Recognition, Machine Translation, and Speech Translation) can be exploited to enhance the model's ability. Specifically, speech and text inputs are firstly fed to different feature extractors to extract acoustic and textual features, respectively. Then, these features are processed by a shared encoder-decoder architecture. We apply several training techniques to improve the performance, including multi-task learning, task-level curriculum learning, data augmentation, etc. Our final system achieves significantly better results than bilingual baselines on supervised language pairs and yields reasonable results on zero-shot language pairs.
\end{abstract}

\section{Introduction}

Multilingual Speech Translation (MultiST) aims to develop a single system that can directly translate speech in different languages into text in many other languages. Due to data scarcity of Speech Translation (ST), multilingual and multimodal models are promising as they enable knowledge transferred from other languages and related tasks like Automatic Speech Recognition (ASR) or Neural Machine Translation (NMT). They also allow zero-shot translation in the settings of no direct parallel data. The IWSLT 2021 MultiST task is held for evaluating the performance under the circumstances. This paper describes our system for the task.

We build a unified model for both speech- and text-related tasks, so that the knowledge from different modalities (speech and text) and different tasks (in this work, the tasks include ST, ASR, and NMT) can be learned together to enhance ST. Specifically, our model consists of three parts - feature extractor, semantic encoder and decoder. For all the tasks, the semantic encoder and the decoder will be shared to learn unified representations. It follows the Transformer (Vaswani et al., 2017) encoderdecoder framework to learn modality-independent features and output text representations. We use the Conv-Transformer (Huang et al., 2020) as feature extractor for speech input, and the word embedding for text input. The extracted features are then fed to the semantic encoder regardless of the input modality.

However, it is difficult for such a unified model to directly digest knowledge from diverse tasks. Therefore, we apply task-level curriculum learning for our model. We presume the ST task is more difficult than the other two tasks (ASR and NMT), as it not only requires acoustic modeling to extract speech representations, but also needs alignment knowledge between different languages for translation. To this end, our training is divided into three steps - ASR and NMT pre-training, joint multitask learning, and ST fine-tuning. What's more, to alleviate the data scarcity problem, we also apply CTC multi-task learning (Graves et al., 2006), data augmentation techniques including SpecAugment (Bahar et al., 2019) and Time Stretch (Nguyen et al., 2020), and knowledge distillation (Liu et al., 2019), etc.

We conduct experiments in the constrained setting, i.e., only the Multilingual TEDx (Salesky et al., 2021) dataset is used for training. It contains speech and transcripts from four languages (Spanish, French, Portuguese, and Italian), and some of them are translated into English and/or other languages of the four mentioned above. Several language pairs for ST are provided without parallel training corpus and evaluated as zero-shot transla- 


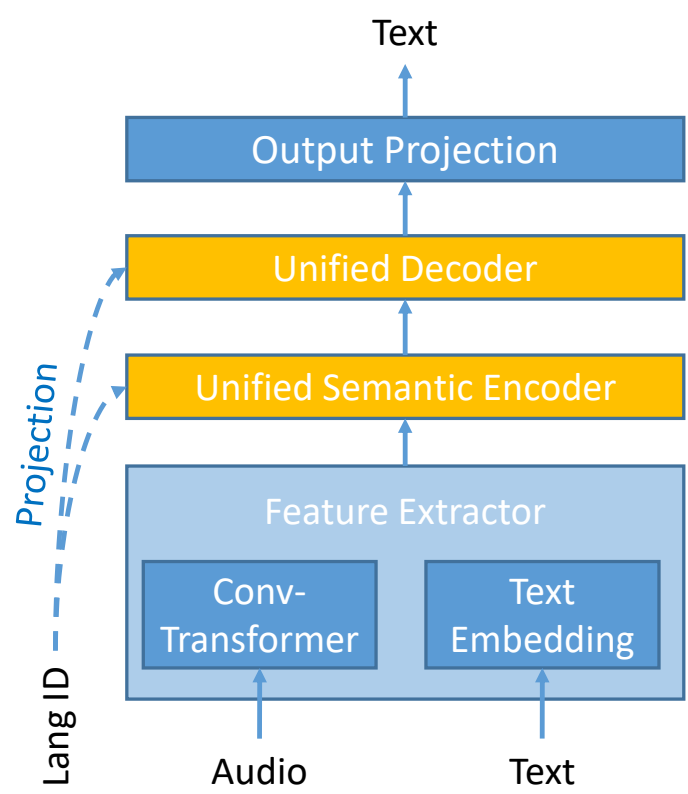

Figure 1: Overall structure of our unified model.

tion. The experimental results show that our unified model can achieve competitive results on both supervised settings and zero-shot settings.

\section{Model Architecture}

The architecture of our unified model, which is based on Transformer (Vaswani et al., 2017), is shown in Figure 1. The NMT part (both input and output is text) follows the basic Transformer setting, i.e. 6 layers for both the encoder and the decoder, each with 8 attention heads, 512 hidden dimensions, and 2048 hidden units in feed-forward layers. For the speech input, we replace the word embedding layer with the Conv-Transformer (Huang et al., 2020) encoder as acoustic modeling to extract audio features, and the rest are shared. The Conv-Transformer encoder gradually downsamples the speech input with interleaved convolution and Transformer layers. We downsample the speech input $8 \times$ times with three Conv-Transformer blocks, each contains three convolution layers (the stride number is 2 in the second convolution layer, and 1 in other layers) and two Transformer layers. The Conv-Transformer is set following Huang et al. (2020) and also consistent with the shared parts (in terms of hidden dimensions, etc). Then, the output is fed into the shared semantic encoder and decoder to produce text representations.

For language encoding, we apply language projection (Luo et al., 2021) to learn language-specific information. It replaces the language embedding in conventional multilingual models with a projec- tion matrix before the positional embedding layer. With language IDs and input modality, our unified model can recognize the task that needs to be completed. For example, our model will perform ASR with speech input and the same language input and output IDs.

\section{Techniques}

Our model is trained in an end-to-end manner with all available data, including the ASR data (speech and transcript) and the ST data (speech, transcript and translation). From the ST data, we also extract the speech-transcript pairs as ASR data, and the transcript-translation pairs as NMT data. We apply task-level curriculum learning to train our model. At the same time, data augmentation, knowledge distillation, and model ensemble are used to further improve the performance. We describe the techniques in details in the rest of this section.

\subsection{Task-Level Curriculum Learning}

As a cross-modal and cross-lingual task, ST is more complicated than ASR or NMT. Therefore, we presume it is better for our unified model to learn in a smoother way. We divide the training procedure into three steps:

1. ASR and NMT pre-training: we use all the ASR and NMT data together to pre-train our unified model with a certain number of steps.

2. Joint multi-task learning: all the data including the ST data are used to jointly train the model in a multi-task manner.

3. ST fine-tuning: we fine-tune the model with only ST data to further improve the performance in specific language pairs ${ }^{1}$.

For all the three steps, we use an additional CTC loss (Graves et al., 2006) on the output of the last layer of Conv-Transformer encoder to assist with the acoustic modeling. What's more, to make the model focus on the ST task, we assign less loss weights to ASR and NMT tasks (both 0.5, while 1.0 for ST) in step 2.

\subsection{Data Augmentation}

We use SpecAugment (Park et al., 2019; Bahar et al., 2019) and Time Stretch (Nguyen et al., 2020) to augment the speech data during training.

\footnotetext{
${ }^{1}$ Note that fine-tuning can only be applied in non zero-shot translation language pairs.
} 
SpecAugment. SpecAugment is a data augmentation technique originally introduced for ASR, but proven to be effective in ST as well. It operates on the input filterbanks and consists of three kinds of operations, time warping, time masking, and frequency masking. We follow Bahar et al. (2019) and only apply time masking and frequency masking. It means that a number of consecutive portions of the speech input are masked in the time or the frequency dimensions. We always apply SpecAugment to both the ASR and ST tasks in the three training steps. We set the parameter for time masking $T$ to 40 and that for frequency masking $F$ to 4 . The number of time and frequency masks applied $m_{T}$ and $m_{F}$ are 2 and 1 , repsectively.

Time Stretch. Time stretching is a kind of augmentation method applied in extracted acoustic features like filterbanks to simulate conventional speed perturbation technique (Ko et al., 2015). Specifically, given a consecutive feature vectors of speech input, it stretches every window of $w$ feature vectors by a factor of $s$ obtained from an uniform distribution of range [low, high]. In this way, some frames are dropped (if $s>1$ ) or repeated (if $s<1$ ) to simulate audio speeding up or down. We only apply Time Stretch in the first two training steps, as we found it does not help much in fine-tuning. We set $w$ to $\infty$, and low $=0.8$, high $=1.25$.

\subsection{Knowledge Distillation}

Teaching the ST model with a pre-trained NMT model using knowledge distillation has been shown effective (Liu et al., 2019). Hence we also use wordlevel knowledge distillation to help with training. Specifically, we minimize the KL divergence between the distribution produced by our model and that produced by the pre-trained NMT model. The tradeoff weight for the knowledge distillation part is set to 0.7 (i.e. 0.3 for cross entropy based on ground-truth targets). We use knowledge distillation only in the ST fine-tuning step.

\subsection{Model Ensemble}

Ensemble decoding is to average the word distribution output from diverse models at each decoding step. It is an very effective approach to improve the quality of NMT models. We select the top 2 or 3 models in terms of BLEU scores on development set for each language pair to perform ensemble decoding. The candidate models are trained with different hyper-parameters.

\begin{tabular}{c|ccccc}
\hline \multirow{2}{*}{ Source } & \multicolumn{5}{|c}{ Target Text } \\
\cline { 2 - 6 } & En & Es & Fr & Pt & It \\
\hline Es & $39 \mathrm{k}(69 \mathrm{~h})$ & $107 \mathrm{k}(189 \mathrm{~h})$ & $7 \mathrm{k}(11 \mathrm{~h})$ & $24 \mathrm{k}(42 \mathrm{~h})$ & $6 \mathrm{k}(11 \mathrm{~h})$ \\
Fr & $33 \mathrm{k}(50 \mathrm{~h})$ & $24 \mathrm{k}(38 \mathrm{~h})$ & $119 \mathrm{k}(189 \mathrm{~h})$ & $16 \mathrm{k}(25 \mathrm{~h})$ & - \\
$\mathbf{P t}$ & $34 \mathrm{k}(59 \mathrm{~h})$ & $\star$ & - & $93 \mathrm{k}(164 \mathrm{~h})$ & - \\
It & $\star$ & $\star$ & - & - & $53 \mathrm{k}(107 \mathrm{~h})$ \\
\hline
\end{tabular}

Table 1: The number of sentences and the duration of audios for the Multilingual TEDx dataset. Same source and target languages mean the ASR data. Noted with $\star$ are the language pairs for zero-shot translation.

\section{Experiments and Results}

\subsection{Experimental Setup}

We only participate in the constrained setting task. Therefore, only the data from the Multilingual TEDx (Salesky et al., 2021) is available. It contains speech and transcripts from four languages (Spanish, French, Portuguese, and Italian), and some of them are translated into other languages of the five (English and the four mentioned above). The data statistics are shown in Table 1.

We use 80-dimensional log-mel filterbanks as acoustic features, which are calculated with $25 \mathrm{~ms}$ window size and $10 \mathrm{~ms}$ step size and normalized by utterance-level Cepstral Mean and Variance Normalization (CMVN). For transcriptions and translations, SentencePiece ${ }^{2}$ (Kudo and Richardson, 2018) is used to generate a joint subword vocabulary with the size of $10 \mathrm{k}$. We share the weights for input and output embeddings, as well as the output projection in CTC module.

Our model is trained with 8 NVIDIA Tesla V100 GPUs, each with a batch size of 32. We use Adam optimizer (Kingma and Ba, 2015) during model training with learning rates selected in $\left\{2 e^{-3}, 1 e^{-3}, 8 e^{-4}, 5 e^{-4}, 3 e^{-4}\right\}$ and warm-up steps selected in $\{2000,6000,10000\}$, followed by the inverse square root scheduler. Dropout rate is selected in $\{0.1,0.2,0.3\}$. We save checkpoints every epoch and average the last 10 checkpoints for evaluation with a beam size of 5 . Our code is based on fairseq S2T ${ }^{3}$ (Wang et al., 2020).

\subsection{Results}

This section shows the results of our unified model in Multilingual TEDx dataset. We display the results of our model for MultiST, as well as ASR and NMT, to show the efficacy of our unified model.

\footnotetext{
${ }^{2}$ https://github.com/google/ sentencepiece

${ }^{3}$ https: / / github.com/pytorch/fairseq/ tree/master/examples/speech_to_text
} 


\begin{tabular}{l|c|c|c|c|c|c|c|c|c|c|c}
\hline Model & Es-En & Es-Fr & Es-Pt & Es-It & Fr-En & Fr-Es & Fr-Pt & Pt-En & Pt-Es $^{\star}$ & It-En $^{\star}$ & It-Es $^{\star}$ \\
\hline Bilingual & 16.60 & 0.70 & 16.16 & 0.50 & 17.49 & 13.74 & 1.26 & 16.83 & - & - & - \\
+ASR data & 19.17 & 9.55 & 29.59 & 14.19 & 24.56 & 25.13 & 23.38 & 21.95 & - & - & - \\
\hline Joint learn & 23.97 & 21.76 & 33.52 & 22.04 & 27.65 & 30.08 & 30.62 & 26.36 & 24.50 & 14.99 & 12.34 \\
Curriculum learn & 25.13 & 22.72 & 35.54 & 24.51 & 29.75 & 31.88 & 31.91 & 28.07 & 26.14 & 15.82 & 14.98 \\
+FT & 25.01 & 22.72 & 35.04 & 24.12 & 29.91 & 31.87 & 31.81 & 27.83 & - & - & - \\
+FT with KD & 25.25 & 23.06 & 35.83 & 24.68 & 30.66 & 32.69 & 32.96 & 28.61 & - & - & - \\
\hline Ensemble & $\mathbf{2 6 . 4 7}$ & $\mathbf{2 3 . 9 4}$ & $\mathbf{3 6 . 5 9}$ & $\mathbf{2 5 . 2 5}$ & $\mathbf{3 1 . 6 0}$ & $\mathbf{3 3 . 8 6}$ & $\mathbf{3 4 . 0 7}$ & $\mathbf{2 9 . 0 2}$ & $\mathbf{2 7 . 1 2}$ & $\mathbf{1 6 . 1 4}$ & $\mathbf{1 6 . 8 2}$ \\
\hline
\end{tabular}

Table 2: BLEU scores of our unified model for Multilingual TEDx test sets. Those marked with ${ }^{\star}$ are the results for zero-shot translation. For each setting, we display the results with highest scores among different hyper-parameters. The ensemble results come from ensembling top 2 or 3 models based on the development sets.

MultiST. Table 2 shows the results of our model on MultiST. The first two rows display the results with only bilingual data. As can be seen, it is difficult for an end-to-end model to produce reasonable results with extremely low resources (less than 30 hours, including language pairs Es-Fr, Es-It and Fr-Pt as in Table 1). With sufficient additional ASR data, all language pairs are improved in a large scale, especially for those low-resource languagepairs (e.g. from 1.26 to 23.38 on Fr-Pt).

The rest rows are the results in multilingual settings, where we use all the available data. "Joint learn" means that we directly train the multilingual model from scratch. "Curriculum learn" displays the results after the first two training steps in Section 3.1, while "+FT" means adding the third finetuning step. "KD" refers to knowledge distillation. We can find that ASR and NMT pre-training helps the model learn better representations to perform translation. Then, fine-tuning with knowledge distillation further improve the results. This indicates the efficacy of our task-level curriculum learning for MultiST. However, we find that fine-tuning only with ground-truth targets would not improve the performance. This might be attributed to the limited ST training data, as all of them are less than 100 hours, which introduces difficulty to learn efficiently. By incorporating knowledge distillation, it enables our model to learn extra meaningful knowledge from NMT, so that it can further improve the results.

It can also be found that our unified model can perform reasonable zero-shot speech translation, as all the zero-shot language pairs achieve higher than 10 BLEU scores. Specifically, results for Pt-Es even achieve similar scores compared with other supervised language pairs. This is mostly because Portuguese and Spanish are similar languages so that it is easier for the model to transfer knowledge from other data.

\begin{tabular}{l|c|c|c|c}
\hline Model & Es & Fr & Pt & It \\
\hline Monolingual & 19.93 & 22.49 & 24.86 & 22.94 \\
Multilingual-ASR & 13.75 & 16.79 & 17.67 & 16.22 \\
Joint learn & 15.69 & 17.46 & 19.85 & 19.12 \\
Curriculum learn & 14.99 & 16.97 & 18.06 & 18.42 \\
$\quad$ +FT & $\mathbf{1 2 . 5 3}$ & $\mathbf{1 4 . 5 6}$ & $\mathbf{1 5 . 7 5}$ & $\mathbf{1 5 . 3 8}$ \\
\hline
\end{tabular}

Table 3: WER of our unified model for ASR test sets.

\begin{tabular}{l|c|c|c|c}
\hline Model & Es-En & Es-Fr & Es-Pt & Es-It \\
\hline Multilingual-NMT & 30.41 & 22.35 & 41.99 & 25.62 \\
Joint learn & 31.11 & $\mathbf{2 8 . 2 5}$ & $\mathbf{4 4 . 1 2}$ & $\mathbf{2 7 . 8 8}$ \\
Curriculum learn & 30.82 & 27.87 & 43.36 & 27.46 \\
+FT & $\mathbf{3 1 . 4 3}$ & 27.81 & 43.53 & 27.46 \\
\hline \hline Model & Fr-En & Fr-Es & Fr-Pt & Pt-En \\
\hline Multilingual-NMT & 35.44 & 36.89 & 37.46 & 33.83 \\
Joint learn & $\mathbf{3 7 . 1 7}$ & $\mathbf{3 9 . 7 8}$ & $\mathbf{4 0 . 6 6}$ & $\mathbf{3 5 . 5 4}$ \\
Curriculum learn & 36.15 & 38.83 & 39.38 & 34.40 \\
+FT & 36.42 & 38.99 & 39.43 & 34.78 \\
\hline
\end{tabular}

Table 4: BLEU of our unified model for NMT test sets.

ASR and NMT. We also test our unified model on the ASR and NMT tasks. Table 3 and Table 4 display the results for ASR and NMT, respectively. "Multilingual-ASR (NMT)" is the model trained only with multilingual ASR (NMT) data. From the results, we can find that ASR also benefits from the task-level curriculum learning procedure. However, it only improves slightly compared to the model only with ASR data, probably because the speech in ST data is sampled from the ASR data (Salesky et al., 2021). It surprises us that NMT can also benefit from extra data from different modality (i.e. speech), although curriculum learning does not improve the performance (probably because we assign less loss weight to NMT task in step 2 as introduced in Section 3.1). This demonstrates the potential of leveraging data from different modalities to train a powerful unified model. Due to the time and data constraint, we leave the exploration into a more powerful unified model with multiple kinds of data as future work. 
Submissions. We submit our results on ST evaluation sets with the ensemble model in Table 2, scoring BLEU scores 35.4 on Es-En, 27.0 on EsFr, 43.2 on Es-Pt, 30.8 on Es-It, 26.7 on Fr-En, 27.0 on Fr-Es, 26.9 on Fr-Pt, 26.7 on Pt-En, 27.0 on Pt-Es, 17.6 on It-En, and 15.4 on It-Es. We also submit our ASR results on evaluation sets with our fine-tuned model (i.e. "+FT" model in Table 3), scoring 11.1 WER on Es ASR, 22.2 on Fr ASR, 16.2 on It ASR, and 23.8 on Pt ASR.

\section{Conclusions}

We present our system submitted to IWSLT 2021 for multilingual speech translation task. In our system, we build a unified transformer-based model to learn the knowledge from different kinds of data. We introduce a task-level curriculum learning procedure to enable our unified model to be trained efficiently. Our results show the efficacy of our unified model to perform multilingual speech translation in both supervised settings and zero-shot settings. Moreover, the results demonstrate the potential of incorporating multilingual and even multi-modal data into one powerful unified model.

\section{References}

Parnia Bahar, Albert Zeyer, Ralf Schlüter, and Hermann Ney. 2019. On using specaugment for end-to-end speech translation. CoRR, abs/1911.08876.

Alex Graves, Santiago Fernández, Faustino J. Gomez, and Jürgen Schmidhuber. 2006. Connectionist temporal classification: labelling unsegmented sequence data with recurrent neural networks. In Machine Learning, Proceedings of the Twenty-Third International Conference (ICML 2006), volume 148 of ACM International Conference Proceeding Series, pages 369-376. ACM.

Wenyong Huang, Wenchao $\mathrm{Hu}$, Yu Ting Yeung, and Xiao Chen. 2020. Conv-transformer transducer: Low latency, low frame rate, streamable end-to-end speech recognition. In Interspeech 2020, 21 st Annual Conference of the International Speech Communication Association, Virtual Event, Shanghai, China, 25-29 October 2020, pages 5001-5005. ISCA.

Diederik P. Kingma and Jimmy Ba. 2015. Adam: A method for stochastic optimization. In 3rd International Conference on Learning Representations, ICLR 2015, San Diego, CA, USA, May 7-9, 2015, Conference Track Proceedings.

Tom Ko, Vijayaditya Peddinti, Daniel Povey, and Sanjeev Khudanpur. 2015. Audio augmentation for speech recognition. In INTERSPEECH 2015, 16th
Annual Conference of the International Speech Communication Association, Dresden, Germany, September 6-10, 2015, pages 3586-3589. ISCA.

Taku Kudo and John Richardson. 2018. SentencePiece: A simple and language independent subword tokenizer and detokenizer for neural text processing. In Proceedings of the 2018 Conference on Empirical Methods in Natural Language Processing: System Demonstrations, pages 66-71, Brussels, Belgium. Association for Computational Linguistics.

Yuchen Liu, Hao Xiong, Jiajun Zhang, Zhongjun He, Hua Wu, Haifeng Wang, and Chengqing Zong. 2019. End-to-end speech translation with knowledge distillation. In Interspeech 2019, 20th Annual Conference of the International Speech Communication Association, Graz, Austria, 15-19 September 2019, pages 1128-1132. ISCA.

Shengjie Luo, Kaiyuan Gao, Shuxin Zheng, Guolin Ke, Di He, Liwei Wang, and Tie-Yan Liu. 2021. Revisiting language encoding in learning multilingual representations. CoRR, abs/2102.08357.

Thai-Son Nguyen, Sebastian Stüker, Jan Niehues, and Alex Waibel. 2020. Improving sequence-to-sequence speech recognition training with on-the-fly data augmentation. In 2020 IEEE International Conference on Acoustics, Speech and Signal Processing, ICASSP 2020, Barcelona, Spain, May 4-8, 2020, pages 76897693. IEEE.

Daniel S. Park, William Chan, Yu Zhang, Chung-Cheng Chiu, Barret Zoph, Ekin D. Cubuk, and Quoc V. Le. 2019. Specaugment: A simple data augmentation method for automatic speech recognition. In Interspeech 2019, 20th Annual Conference of the International Speech Communication Association, Graz, Austria, 15-19 September 2019, pages 2613-2617. ISCA.

Elizabeth Salesky, Matthew Wiesner, Jacob Bremerman, Roldano Cattoni, Matteo Negri, Marco Turchi, Douglas W. Oard, and Matt Post. 2021. The multilingual tedx corpus for speech recognition and translation. CoRR, abs/2102.01757.

Ashish Vaswani, Noam Shazeer, Niki Parmar, Jakob Uszkoreit, Llion Jones, Aidan N. Gomez, Lukasz Kaiser, and Illia Polosukhin. 2017. Attention is all you need. In Advances in Neural Information Processing Systems 30: Annual Conference on Neural Information Processing Systems 2017, December 4-9, 2017, Long Beach, CA, USA, pages 5998-6008.

Changhan Wang, Yun Tang, Xutai Ma, Anne Wu, Dmytro Okhonko, and Juan Pino. 2020. Fairseq S2T: Fast speech-to-text modeling with fairseq. In Proceedings of the 1st Conference of the Asia-Pacific Chapter of the Association for Computational Linguistics and the 10th International Joint Conference on Natural Language Processing: System Demonstrations, pages 33-39, Suzhou, China. Association for Computational Linguistics. 